\title{
Evaluation of immune responses and protection induced by A2 and nucleoside hydrolase (NH) DNA vaccines against Leishmania chagasi and Leishmania amazonensis experimental infections
}

\author{
Francisca H.C. Zanin ${ }^{\text {a }}$, Eduardo A.F. Coelho ${ }^{\text {b,c }}$, Carlos A.P. Tavares ${ }^{\text {c }}$, \\ Eduardo A. Marques-da-Silva ${ }^{d}$, Miriam Maria Silva Costa ${ }^{c}$, Simone A. Rezende ${ }^{d}$, \\ Ricardo T. Gazzinelli ${ }^{c}$, Ana Paula Fernandes ${ }^{\text {a,* }}$ \\ ${ }^{a}$ Faculdade de Farmácia, Universidade Federal de Minas Gerais, Avenida Antônio Carlos, \\ 6627, 31270-901 Belo Horizonte, Minas Gerais, Brazil \\ ${ }^{\mathrm{b}}$ Setor de Patologia Clínica, Coltec, Universidade Federal de Minas Gerais, Avenida Antônio Carlos, 6627, \\ 31270-901 Belo Horizonte, Minas Gerais, Brazil \\ ${ }^{\mathrm{c}}$ Departamento de Bioquímica e Imunologia, Instituto de Ciências Biológicas, Universidade Federal de Minas Gerais, \\ Avenida Antônio Carlos, 6627, 31270-901 Belo Horizonte, Minas Gerais, Brazil \\ ${ }^{\mathrm{d}}$ Escola de Farmácia, Universidade Federal de Ouro Preto, Ouro Preto, Minas Gerais, Brazil
}

Received 5 August 2006; accepted 3 May 2007

Available online 16 May 2007

\begin{abstract}
Several antigens have been tested as vaccine candidates against Leishmania infections but controversial results have been reported when different antigens are co-administered in combined vaccination protocols. Immunization with A2 or nucleoside hydrolase (NH) antigens was previously shown to induce Th1 immune responses and protection in BALB/c mice against Leishmania donovani and L. amazonensis (A2) or L. donovani and L. mexicana $(\mathrm{NH})$ infections. In this work, we investigated the protective efficacy of A2 and NH DNA vaccines, in $\mathrm{BALB} / \mathrm{c}$ mice, against L. amazonensis or L. chagasi challenge infection. Immunization with either A2 (A2-pCDNA3) or NH (NH-VR1012) DNA induced an elevated IFN- $\gamma$ production before infection; however, only A2 DNA immunized mice were protected against both Leishmania species and displayed a sustained IFN- $\gamma$ production and very low IL-4 and IL-10 levels, after challenge. Mice immunized with NH/A2 DNA produced higher levels of IFN- $\gamma$ in response to both specific recombinant proteins (rNH or rA2), but displayed higher IL-4 and IL-10 levels and increased edema and parasite loads after L. amazonensis infection, as compared to A2 DNA immunized animals. These data extend the characterization of the immune responses induced by $\mathrm{NH}$ and $\mathrm{A} 2$ antigens as potential candidates to compose a defined vaccine and indicate that a highly polarized type 1 immune response is required for improvement of protective levels of combined vaccines against both $L$. amazonensis and L. chagasi infections.
\end{abstract}

(C) 2007 Elsevier Masson SAS. All rights reserved.

Keywords: DNA vaccine; A2 antigen; Leishmania chagasi; Leishmania amazonensis

\footnotetext{
* Corresponding author. Tel.: +55 313499 6884; fax: +55 3134996985.

E-mail addresses: anav@uai.com.br, anapaula.fernandes@pesquisador. cnpq.br (A.P. Fernandes).
}

\section{Introduction}

Infection by parasites of the genus Leishmania induces a wide spectrum of diseases. In Americas, at least eight different species of Leishmania are major ethiologic agents of leishmaniasis, including Leishmania chagasi and L. amazonensis, which have wide and overlapping areas of transmission and 
are associated with visceral (VL) and tegumentary (TL) leishmaniasis, respectively [1,2].

Due to the high antigenic diversity of the Leishmania parasites, a multistage vaccine may be necessary for optimal protection against infections. Although several defined antigenic preparations have been tested as vaccine candidates against Leishmania infections, few were shown to induce protection against more than one Leishmania species. In addition, distinct results have been reported when different antigens are coadministered in the same vaccination protocol [3-6].

The A2 protein was first identified in L. donovani as part of an amastigote stage-specific protein family [7]. Karyotype analysis revealed that $\mathrm{A} 2$ genes are conserved in $L$. donovani, L. chagasi, L. amazonensis and L. mexicana species [8]. Immunization with recombinant $\mathrm{A} 2(\mathrm{rA} 2)$ protein or A2 DNA was shown to protect $\mathrm{BALB} / \mathrm{c}$ mice against $L$. amazonensis [5] or L. donovani infection $[9,10]$.

The nucleoside hydrolase $(\mathrm{NH})$ antigen was initially identified in L. donovani as a major fraction of the fucose-mannose ligand (FML) antigenic complex [11]. This surface glycoprotein complex is expressed in L. donovani, L. chagasi, $L$. amazonensis and L. major [12]. Paraguai-de-Souza et al. [13] demonstrated that immunization with recombinant $\mathrm{NH}$ $(\mathrm{rNH})$ protein was able to induce partial protection in $\mathrm{BALB} / \mathrm{c}$ mice against $L$. donovani infection. Partial protection was also obtained by immunization with NH DNA in BALB/c mice against $L$. chagasi or L. mexicana infections [14].

Based on previous evidences that $\mathrm{A} 2$ and $\mathrm{NH}$ antigens induce $\mathrm{Th} 1$ immune responses and partial protection against Leishmania species, in this work, we investigated the immune responses and protective efficacy of A2 (A2-pCDNA3) and $\mathrm{NH}$ (NH-VR1012) DNA and their association (NH/A2 DNA), in BALB/c mice, against $L$. chagasi or L amazonensis infections. DNA vaccines were chosen as an antigenic delivery system, since they are easily prepared and able to induce type 1 and $\mathrm{CD}^{+}$cytotoxic T-lymphocyte immune responses $[15,16]$.

\section{Materials and methods}

\subsection{Parasites}

Leishmania chagasi (MHOM/BR/1975/M2682) was grown at $26{ }^{\circ} \mathrm{C}$ in Dulbecco Modified Eagle medium (DMEM, Sigma), supplemented with $20 \%$ heat-inactivated fetal bovine serum (FSB, Cultilab, Brazil), $2 \mathrm{mM}$ L-glutamine, $25 \mathrm{mM}$ HEPES, $50 \mu \mathrm{M} 2$ 2-mercaptoetanol and $20 \mu \mathrm{g} / \mathrm{ml}$ of gentamicin, at $\mathrm{pH}$ 7.0. L. amazonensis (IFLA/BR/1967/PH-8) was grown at $23{ }^{\circ} \mathrm{C}$ in Schneider's medium (Sigma) supplemented with $20 \%$ FSB, $20 \mathrm{mM}$ L-glutamine, $50 \mu \mathrm{g} / \mathrm{ml}$ of gentamicin, $200 \mathrm{U} / \mathrm{ml}$ of penicillin and $100 \mu \mathrm{g} / \mathrm{ml}$ of streptomycin, at $\mathrm{pH}$ 7.4 .

\subsection{Preparation of antigens}

Soluble L. amazonensis Antigenic extract (SLA) was prepared from stationary phase promastigotes, as described previously [5]. Briefly, $2 \times 10^{8}$ promastigotes $/ \mathrm{ml}$ were collected and resuspended in $10 \mathrm{ml}$ of sterile phosphate buffer saline (PBS). After five freezing-thawing cycles, the suspension was centrifuged at $8000 \times g$ for $30 \mathrm{~min}$ at $4{ }^{\circ} \mathrm{C}$. The supernatant was collected, quantified by the Bradford method [17] and stored at $-86^{\circ} \mathrm{C}$.

L. chagasi promastigotes antigenic extract (LcPA) was prepared as described [18]. Briefly, $2 \times 10^{8}$ promastigotes $/ \mathrm{ml}$ of L. chagasi were harvested from late log phase cultures by centrifugation, washed three times in sterile PBS, disrupted by three rounds of freezing-thawing and five cycles $(30 \mathrm{~s}$ each one) of sonication. The suspension were centrifuged at $8000 \times g$ for $30 \mathrm{~min}$ at $4{ }^{\circ} \mathrm{C}$ and the supernatant was collected, quantified by Bradford method [17] and stored at $-86^{\circ} \mathrm{C}$.

\subsection{Purification of plasmids}

pCDNA3-A2 (A2 DNA) and pET16b-A2 plasmids were kindly provided by Dr. Greg Matlashewski (Microbiology Immunology Department, McGill University, Montreal, Quebec, Canada) and VR1012-NH (NH DNA) and pMALc2-NH by Dr. Clarisa Palatnik de Sousa (Departamento de Microbiologia, Universidade Federal do Rio de Janeiro, Rio de Janeiro, Brazil). Escherichia coli DH5 $\alpha$ cells transformed with A2 DNA, NH DNA or with empty plasmids (pCDNA3 or VR1012) were cultured in Luria Bertani (LB; Gibco) liquid medium containing ampicilin $(100 \mu \mathrm{g} / \mathrm{ml})$. Endotoxin-free plasmids DNA were isolated using maxipreps DNA purification kits (Qiagen). Plasmids concentrations were determined by spectrophotometry $(260: 280 \mathrm{~nm})$, using the Gene Quant II apparatus (Pharmacia Biotech).

\subsection{Purification of recombinant proteins}

pET16b-A2 plasmid was utilized for expression and purification of recombinant $\mathrm{A} 2(\mathrm{rA} 2 ; \sim 53 \mathrm{kDa})$ protein, as previously described [19]. The recombinant $\mathrm{NH}(\mathrm{rNH})$ protein expression was obtained after excision of its encoding sequence from pMALc2-NH plasmid, using EcoRI and XbaI endonucleases and ligation into pPROEX-b vector. Then, $E$. coli DH5 $\alpha$ cells were transformed with pPROEX b-NH and grown in the presence of $1 \mathrm{mM}$ of IPTG for $3 \mathrm{~h}$ (Promega, Montreal, Canada). Cells were disrupted by five cycles of freezing-thawing, followed by mild ultrasonic treatment (five cycles of $30 \mathrm{~s}$ each one with an ultrasonic processor) and then were centrifuged at $13,000 \times g$ for $30 \mathrm{~min}$ at $4{ }^{\circ} \mathrm{C}$. After protein expression, recombinant NH (NH-HIS $\sim 36 \mathrm{kDa}$ ) protein was purified by nickel affinity chromatography, according to the manufacturer's instructions (Qiagen).

\subsection{Mice and immunization protocol}

Groups of female BALB/c mice $(n=8), 4-6$ weeks old, were immunized intramuscularly in their left hind with $100 \mu \mathrm{g}$ of A2 DNA, NH DNA or with NH/A2 DNA (100 $\mu \mathrm{g}$ of each plasmid). Two plasmid doses were administered in PBS plus sucrose $25 \%$, at 3-weeks intervals. Control mice 
received $100 \mu \mathrm{g}$ of either pCDNA3 or VR1012 vectors alone or in combination (100 $\mu \mathrm{g}$ each), in PBS plus sucrose $25 \%$ or only PBS plus sucrose $25 \%$.

\subsection{Challenge infection}

Four weeks after the last vaccine dose, BALB/c mice were infected in the tail vein with $1 \times 10^{7}$ late $\log$ phase $L$. chagasi promastigotes. Mice were sacrificed 35 days after challenge, when liver and spleen were harvested for determination of parasite burdens and cytokine responses.

For L. amazonensis infection, mice were challenged with $1 \times 10^{6}$ late stationary phase promastigotes into their right hind footpad. In these animals, the course of disease was monitored at weekly intervals, by measuring footpad thickness with a metric caliper. Results were expressed as the increase in the thickness of the infected hind foot compared to the uninfected left foot. After lesion onset, the course of infection was monitored during 9 weeks, when tissue skin fragments were harvested for parasite burden determinations and spleen cells were collected for evaluation of cellular immune responses.

\subsection{Parasite quantification}

Liver and spleen were collected to determine the parasite burdens in mice challenged with $L$. chagasi, as previously described [18]. Briefly, organs were weighted, fragmented and a tissue homogenate was obtained in $1 \mathrm{ml}$ of DMEM plus $20 \%$ FBS, at $\mathrm{pH}$ 7.0. Five-fold serial dilutions were performed in 96-well flat-bottom microtiter plates (Nunc, Nunclon), in duplicate, and plates were incubated at $23{ }^{\circ} \mathrm{C}$. Plates were evaluated for parasite growth using a microscope (Axiovert 25 , Zeiss). The number of viable parasites was determined from the highest dilution at which promastigotes had grown, after 12 days of incubation

The number of viable parasites at the site of L. amazonensis infection was determined by a limiting dilution assay, as described previously [5]. Briefly, skin fragments were excised and homogenized in Schneider's medium supplemented with $20 \%$ FBS, at $\mathrm{pH}$ 7.4. Each tissue homogenate was serially diluted in 96-well plates (Nunc, Nunclon), in duplicate, and incubated at $23{ }^{\circ} \mathrm{C}$. The wells containing motile promastigotes were identified and the number of viable parasites was determined from the highest dilution at which promastigotes had grown after 7 days of incubation.

\subsection{Cytokine production assays}

Splenocyte cultures were performed as described previously [5]. Briefly, single-cell preparations $\left(5 \times 10^{6}\right.$ cells $\left./ \mathrm{ml}\right)$ obtained from spleen were plated, in duplicate, in 24-well plates (Nunc, Nunclon). Cells were incubated in DMEM (background control) or separately stimulated with concanavalin A (positive control; $5 \mu \mathrm{g} / \mathrm{ml}$ ), rA2 or rNH proteins (10 $\mu \mathrm{g} /$ $\mathrm{ml})$, SLA or LcPA $(50 \mu \mathrm{g} / \mathrm{ml})$ in $5 \% \mathrm{CO}_{2}$, for $48 \mathrm{~h}$, at $37^{\circ} \mathrm{C}$. Levels of IFN- $\gamma$, IL-4 and IL- 10 were assessed in supernatants by ELISA, as described elsewhere [5,20].

\subsection{Statistical analysis}

All data comparisons were tested for significance by using unpaired Student's $t$-test or Kruskal-Wallis test. Differences were considered statistically significant when $P$ values were $<0.05$.

\section{Results}

\subsection{Immunization with A2 DNA, but not NH DNA, confers protection in $B A L B / c$ mice against $\mathrm{L}$. chagasi or L. amazonensis}

The protective effect of immunization of $\mathrm{BALB} / \mathrm{c}$ mice with $\mathrm{NH}, \mathrm{A} 2$ or NH/A2 DNA vaccines against $L$. chagasi infection was evaluated by measuring parasite loads in liver and spleen (Fig. 1). Non-significant reductions in parasite numbers in liver or spleen were observed in mice immunized with NH DNA, when compared to saline or VR1012 immunized animals. In contrast, mice immunized with A2 DNA or NH/A2DNA displayed significant reductions in both hepatic and splenic parasite loads, as compared to saline, pCDNA3, VR1012/pCDNA3 and NH groups. Non-significant differences were observed between $\mathrm{A} 2$ and $\mathrm{A} 2 / \mathrm{NH}$ groups.

After L. amazonensis challenge infection, the protective efficacy of immunization was evaluated by measuring lesion development (Fig. 2A) and parasite loads in the infected footpads (Fig. 2B). No significant reduction in footpad swelling or parasite loads was observed in animals immunized with $\mathrm{NH}$ DNA, as compared to saline or VR1012 immunized animals. In contrast, mice immunized with A2 DNA showed decreased edema of the infected footpads as compared to saline or pCDNA3 groups (Fig. 2A). The reduction in lesion development observed in A2 DNA group correlated with a $10^{6}$ decrease in parasite burden in the infected footpads as compared to saline or pCDNA3 (Fig. 2B). Mice immunized with NH/A2 DNA also showed significant reductions in footpad

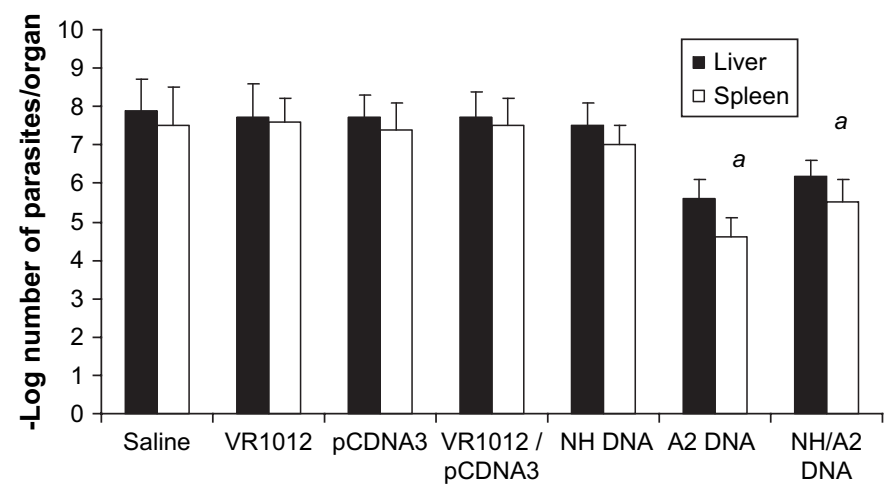

Fig. 1. Leishmania chagasi protection assays. Mice were vaccinated as described in Section 2 and challenged i.v. with $1 \times 10^{7}$ L. chagasi promastigotes. Parasite burden determinations were performed by limiting dilution assays, 35 days after infection. Experiments were performed in triplicate. Each bar represents mean \pm standard deviation of results obtained from four mice per group. The letter in italic (a) indicates significant differences as compared to saline and control DNA groups (pCDNA3, VR1012 or VR1012/pCDNA3). 

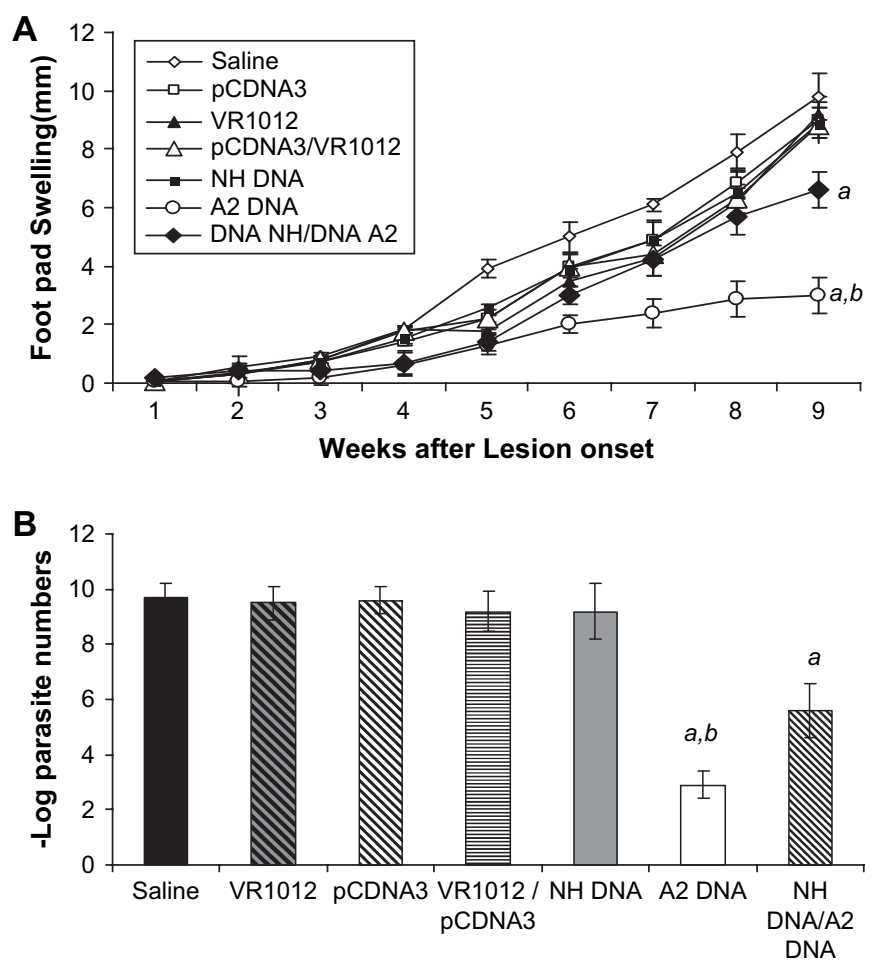

Fig. 2. Leishmania amazonensis protection assays. Mice were vaccinated, as described in Section 2.5 and challenged with $1 \times 10^{6}$ late stationary phase L. amazonensis promastigotes. Panel (A) shows lesion development in immunized groups, monitored weekly with a caliper. Panel (B) shows parasite loads per mg of tissue fragments, as determined by a limiting dilution assays, 9 weeks post-challenge. Experiments were performed in triplicate. Each point or bar represents the mean \pm standard deviation of results obtained from four mice per group. Italic letters indicate significant differences as compared to saline and control DNA groups (pCDNA3, VR1012 or VR1012/pCDNA3) (a) or as compared to the NH/A2 DNA group $(b)$.

swelling and parasite loads in comparison to saline or VR1012/pCDNA3 groups; however, both lesion size and parasite loads were significantly higher as compared to A2 DNA group.

\subsection{Characterization of the cellular (IFN- $\gamma, I L-4$ and $I L-10)$ response in immunized animals, before challenge infection}

The production of IFN- $\gamma$, IL-4 and IL-10 by spleen cells of immunized mice was evaluated prior to (30-days after second vaccine dose) and after challenge infection (35-days after $L$. chagasi infection and 9 weeks after L. amazonensis infection).

Prior to challenge infection (Fig. 3A), spleen cells taken from animals immunized with either NH DNA, A2 DNA or $\mathrm{NH} / \mathrm{A} 2$ DNA produced high levels of IFN- $\gamma$ in response to each recombinant protein, in comparison to saline or control DNA groups (pCDNA3, VR1012 or VR1012/pCDNA3); however, mice immunized with A2 DNA produced significantly higher levels of this cytokine as compared to NH DNA group, after specific recombinant protein stimulation. Mice immunized with NH DNA showed a significantly higher IL-4 production (Fig. 3B), in comparison to the other groups. IL-10 levels were not significantly different among the different groups, except for NH DNA group which showed an increased production of this cytokine in response to $\mathrm{rNH}$ stimulation.

\subsection{Characterization of the cellular $(I F N-\gamma, I L-4$ and $I L-$ 10) response in immunized mice, after challenge infection with L. chagasi or L. amazonensis}

After $L$. chagasi challenge infection, lower IFN- $\gamma$ levels (Fig. 4A) and significantly higher IL-4 and IL-10 levels (Fig. 4B) were detected in response to LcPA stimulation, in mice that received saline or control DNA, as compared to the levels produced before challenge. In contrast, immunization with A2 DNA induced a higher IFN- $\gamma$ production and lower IL-4 and IL-10 levels in response to both rA2 and LcPA as compared to saline, control DNA groups or NH DNA (after stimulation with $\mathrm{NH}$ or LcPA). Although splenocytes from NH/A2 DNA immunized animals produced increased IFN- $\gamma$ levels (Fig. 4A) in response to rNH, rA2 or LcPA stimulation, these animals also produced significantly higher IL-4 and IL-10 levels (Fig. 4B), as compared with A2 DNA immunized mice. These data indicate that, after $L$. chagasi infection the IFN- $\gamma$ response was boosted in NH/A2 DNA immunized animals but this was not sufficient to significantly improve the control of parasite replication, when compared to the levels observed in mice immunized with A2.

After L. amazonensis challenge infection, mice that received saline or control DNA produced low levels of IFN- $\gamma$ (Fig. 4C) and higher IL-4 and IL-10 levels (Fig. 4D) in response to SLA, as compared to those observed before challenge. Splenocytes of mice immunized with NH DNA showed a similar cytokine profile. Moreover, IFN- $\gamma$ production in response to rNH or SLA was not significantly boosted after infection in the NH DNA immunized mice. Mice immunized with A2 DNA, in contrast, showed a significant and sustained IFN- $\gamma$ production and very low IL-4 and IL-10 levels as compared to the levels obtained prior to infection. Significantly higher IFN- $\gamma$ levels and lower IL-4 and IL-10 in response to $\mathrm{rA} 2$ or SLA were also observed in these mice as compared to saline and pCDNA3 groups. A similar profile was observed in NH/A2 mice immunized; however these mice produced lower IFN- $\gamma$ and higher IL-4 and IL10 levels in response to $\mathrm{rNH}, \mathrm{rA} 2$ or SLA stimulation, as compared to A2 immunized animals.

\section{Discussion}

Protective immunity against $L$. chagasi and L amazonensis is dependent of IFN- $\gamma$ production, a marker of type 1 immune response [3,20-23]. In agreement, the significant reduction of parasite loads observed in mice immunized with A2 DNA in skin (L. amazonensis challenge) or in liver and spleen ( $L$. chagasi challenge) was associated with high IFN- $\gamma$ and low IL-4 and IL-10 production, before and after challenge infection. Similar findings were previously reported indicating that immunization with A2 antigen is protective against $L$. donovani and L. amazonensis [5,9,10]. 

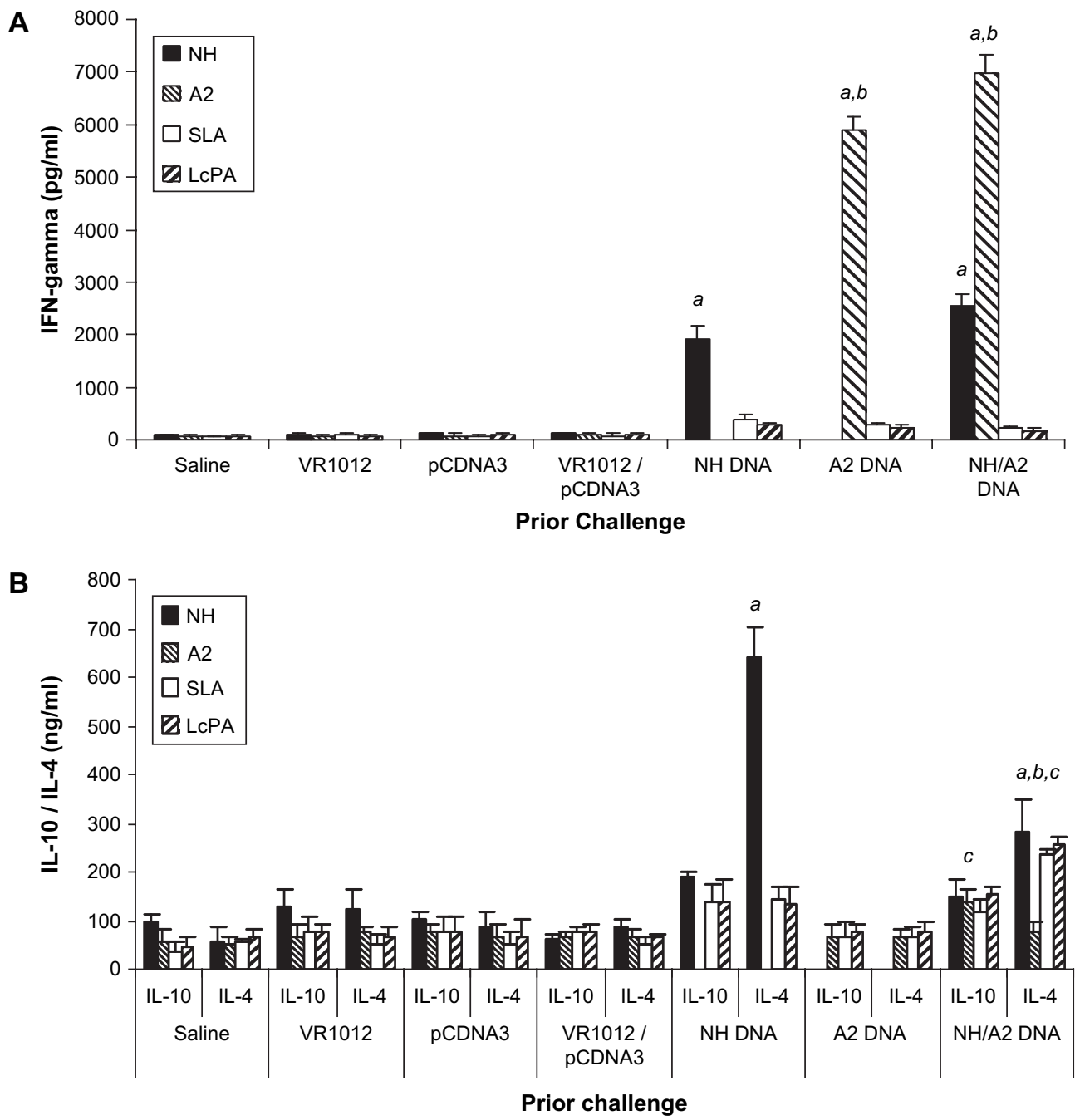

Fig. 3. Production of IFN- $\gamma$, IL-4 and IL-10 by spleen cells prior challenge infections. Panel A shows IFN- $\gamma$ levels and panel B IL-4 and IL-10 levels, as determined by ELISA in culture supernatants of spleen cells stimulated with rNH or rA2 proteins ( $10 \mu \mathrm{g})$, SLA or LcPA ( $50 \mu \mathrm{g})$. Each bar represents mean \pm standard deviation of results obtained from four mice per group. Letters in italic indicate statistical significant differences as compared to saline and control DNA groups (pCDNA3, VR1012 or VR1012/pCDNA3) (a), as compared to NH DNA group $(b)$ or as compared to A2 DNA immunized group (c).

Mice immunized with NH DNA were not protected against L. chagasi, since significant reduction on parasite burden in liver and spleen were not detected. Partial protection in $\mathrm{BALB} / \mathrm{c}$ mice immunized with $\mathrm{rNH}$ protein or NH DNA and challenged with $L$. donovani or L. chagasi was previously reported by Paraguai-de-Souza et al. [13] and Aguilar-Be et al. [14], respectively. Differences in immunization protocols and virulence of parasites might explain these different results.

Significant reductions of parasite numbers at both spleen and liver were observed in A2 immunized mice. The evaluation of parasite loads in the spleen, although frequently neglected in vaccine studies, might be an important marker of vaccine efficacy against visceral leishmaniasis, since the requirements for effective immune mechanisms may not be adequately induced by vaccination in the specific tissue sites. Moreover, during the early stages of visceral infection in $\mathrm{BALB} / \mathrm{c}$ mice, parasites multiply in large numbers in the liver. Lately, the hepatic parasite load tends to decrease while the splenic parasitism tends to increase $[21,24,25]$. Thus, it seems that liver serves as an indicator of the initial multiplication of parasites and spleen as a reservoir for them [25].

After $L$. amazonensis infection, the IFN- $\gamma$ production primed by NH DNA immunization was not boosted. The lack of a boost effect may not be attributed, however, to the lack of $\mathrm{NH}$ expression by L. amazonensis, since anti-NH antibodies present in sera of NH DNA immunized mice reacted with $\mathrm{NH}$ native protein in antigenic preparations of $L$. amazonensis or L. chagasi (data not shown). However, before challenge, $\mathrm{NH}$ immunized animals displayed increased levels of IL-4 in response to NH stimulation, as compared to saline controls. After challenge, an increased production of both IL-4 and IL-10 in response to NH or SLA was observed in these animals, when compared to A2 immunized mice. Thus, the failure of NH DNA immunized mice to control L. amazonensis infection might be attributed to the higher IL-4 and IL-10 levels observed in these animals, when compared to the levels observed in A2 immunized mice. 


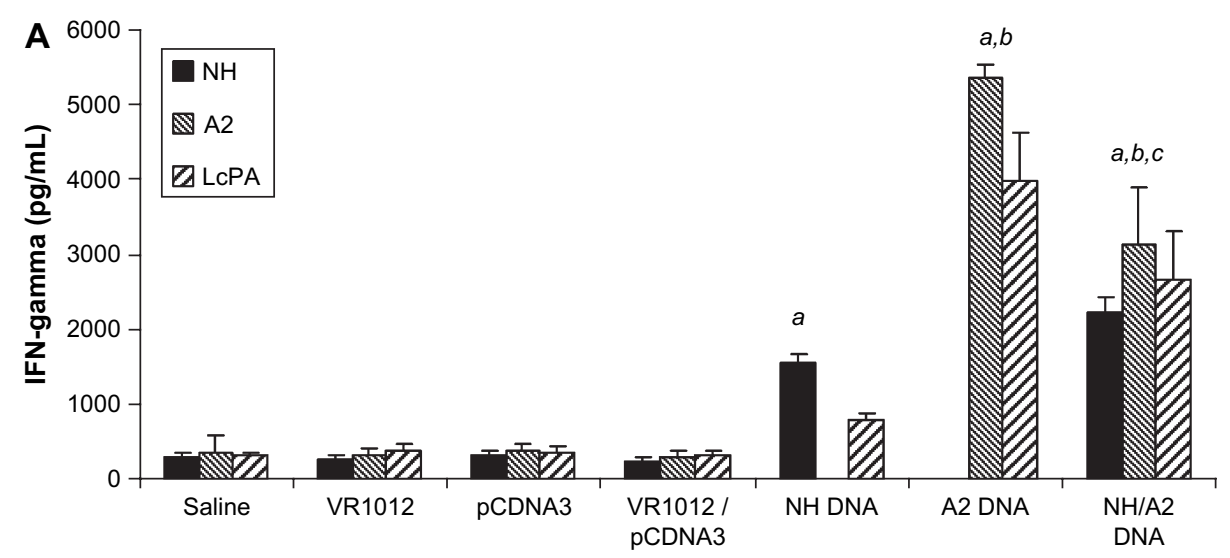

After L.chagasi infection

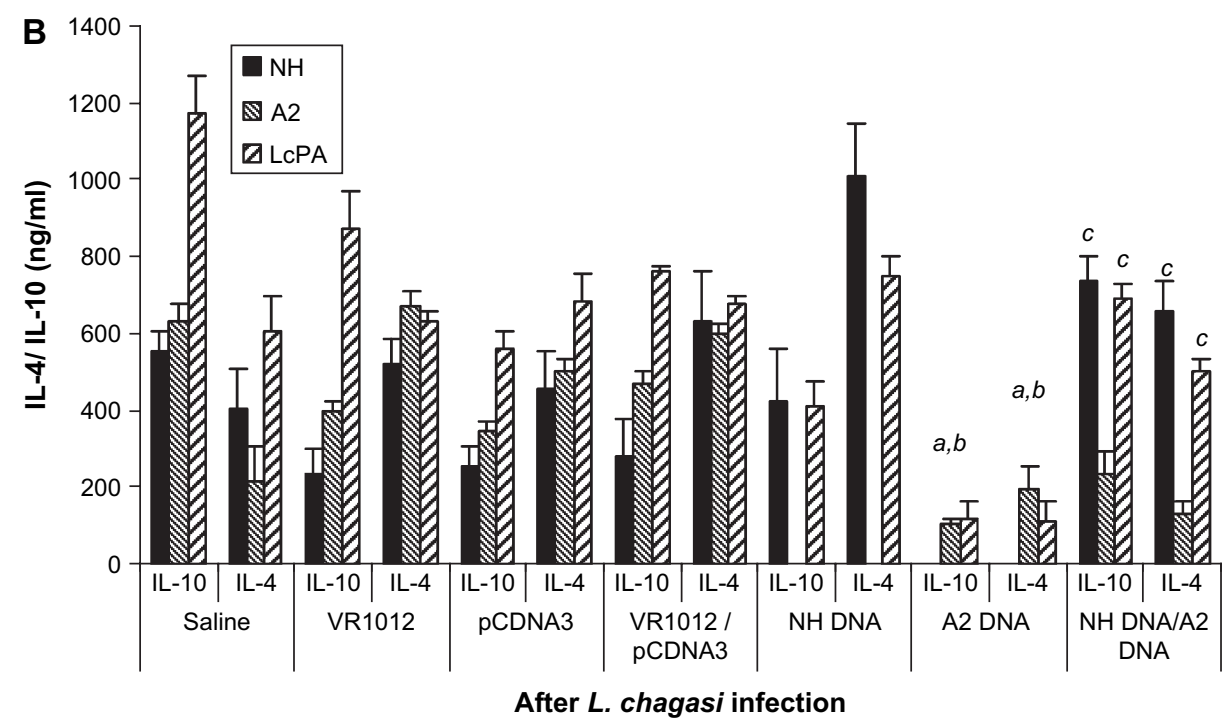

Fig. 4. Production of IFN- $\gamma$, IL-4 and IL-10 by spleen cells 35 -days after $L$. chagasi or 9 weeks after $L$. amazonensis challenge infections. IFN- $\gamma$, IL-4 and IL-10 were determined by ELISA in culture supernatants of spleen cells stimulated with rNH or rA2 proteins (10 $\mu \mathrm{g})$, SLA or LcPA (50 $\mu \mathrm{g})$. Each bar represents mean \pm standard deviation of results obtained from four mice per group. Panels A and C show IFN- $\gamma$ levels after L. chagasi or L. amazonensis infections, respectively. Panels B and D show IL-4 and IL-10 levels after L. chagasi or L. amazonensis infections, respectively. Italic letters indicate statistical significant differences as compared to saline and control DNA groups (pCDNA3, VR1012 or VR1012/pCDNA3) (a), as compared to NH DNA group $(b)$ or as compared to A2 DNA group (c).

Recent evidence suggest, that in L. amazonensis experimental infection, combined effects of low levels of IFN- $\gamma$ and high levels of parasite-specific antibodies promote the rapid recruitment of immature or insufficiently activated macrophages, that favors the replication of amastigotes and progression of disease [20]. IL-4 and IL-10 contributes partially to these deficient immune responses [23,27]. IL-10 has multiple effects in suppressing microbicidial leishmaniasis activity in the macrophages, IFN- $\gamma$ production and, consequently, preventing parasite clearance in susceptible mice $[21,22,25,28,29]$.

Although decreased edema and parasite loads were observed in NH/A2 DNA immunized mice as compared to control groups, the association of NH DNA and A2 DNA partially inhibited the A2 DNA protective effect. Similar results were observed in mice immunized with recombinant A2 and LACK proteins [5]. The IFN- $\gamma$ levels induced by A2 were completed inhibited in A2/LACK immunized animals, suggesting that an antigenic competition mechanism would be involved. The failure to induce protection by immunization with LACK antigen was also accompanied by high levels of IL-4 and IL-10 [5,18]. Since NH/A2 immunized mice were still able to produce significant amounts of IFN- $\gamma$ in response to rA2, rNH, LcPA or SLA, it seems that the inhibition observed is not due to antigenic competition, but might be attributed to the high IL-4 and IL-10 levels. Stober et al. [29] demonstrated that pre-challenge high IL10 production was associated with failure of rLACK protein to protect $\mathrm{BALB} / \mathrm{c}$ mice against $L$. major infection and suggested that the ratio of IFN- $\gamma / \mathrm{IL}-10$ levels may provide an indicator of vaccine success in this murine model. IL-10 has been shown to participate of parasite persistent in spleen of $L$. donovani infected mice. Mixed immune responses with concomitant production of IFN- $\gamma$, IL- 4 and IL-10 are also observed in $L$. donovani infections and the progressive development of splenic pathology was associated with high levels of both TNF and interleukin IL-10. IL-10 promotes impaired DC migration into T-cell areas with consequent ineffective Tcell priming [30]. 


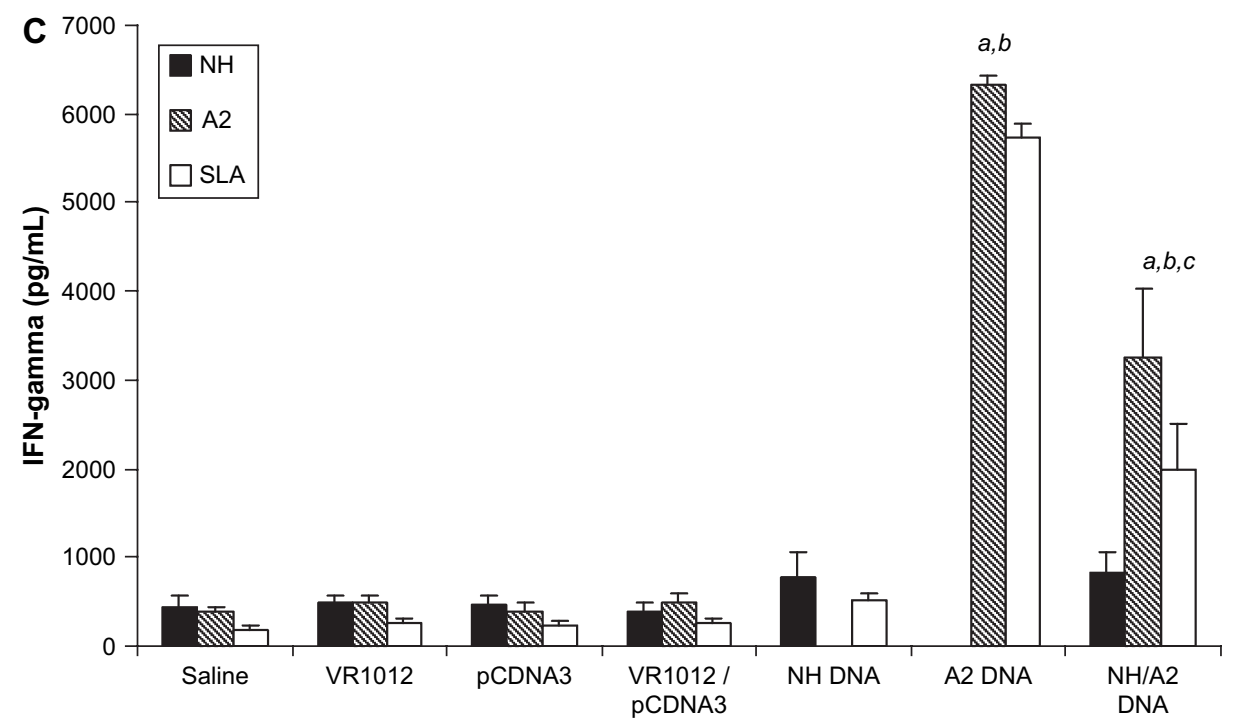

After $L$. amazonensis infection

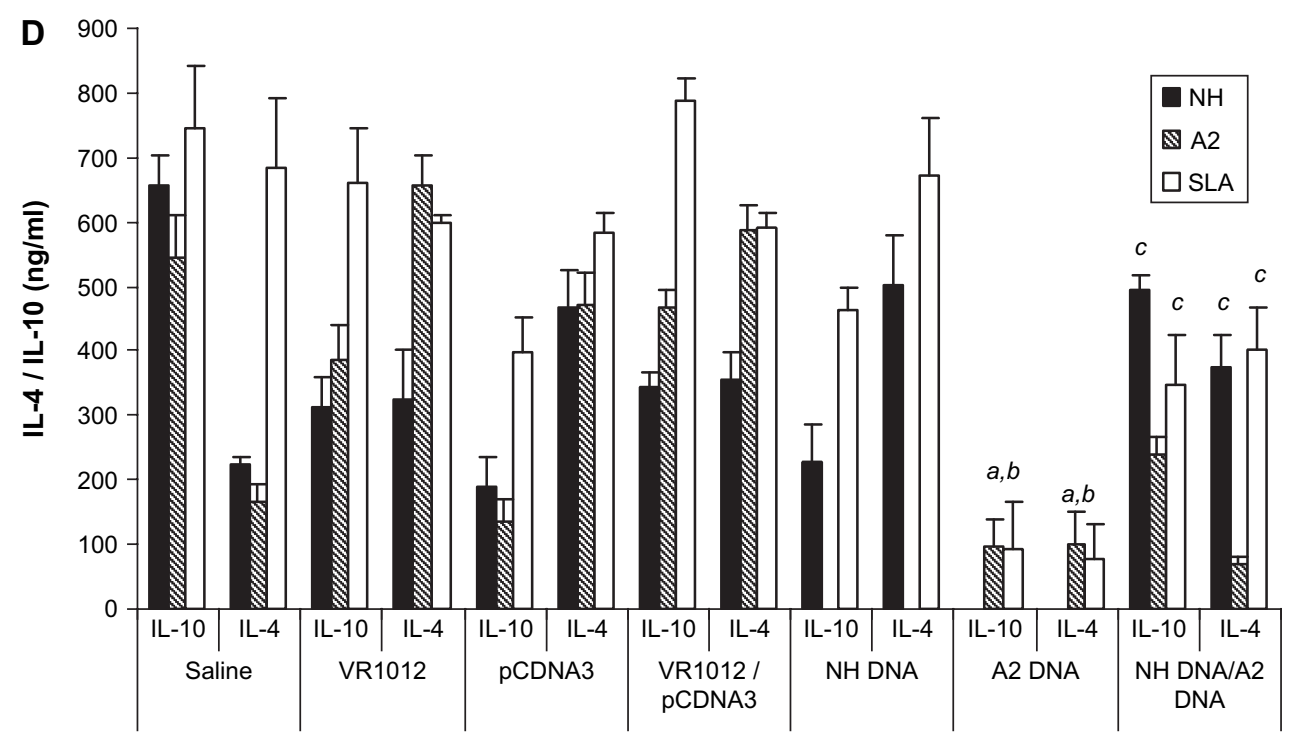

After $L$. amazonensis infection

Fig. 4. (Continued)

Taken together, our data confirm that high IFN $-\gamma$ and low IL-4 and IL-10 levels are required for protection in BALB/c mice against $L$. chagasi and $L$. amazonensis infection. The association of well-characterized antigens and potent adjuvant systems, such as viral expression vectors might achieve this polarized type 1 immune response. In this way, results presented herein extended the characterization of the immune responses induced by $\mathrm{NH}$ and $\mathrm{A} 2$ antigens as potential candidates to compose a vaccine applicable to the complex epidemiological context of leishmaniasis in Americas.

\section{Acknowledgements}

Authors thank Dr. Greg Matlashewski (Department of Microbiology and Immunology, McGill University, Montreal,
Quebec, Canada) and Dr. Clarisa Palatnik de Sousa (Department of Microbiology, Universidade Federal do Rio de Janeiro, Rio de Janeiro, Brazil) for provided the pCDNA3-A2 and pET16b-A2; VR1012-NH and pMALc2-NH plasmids, respectively. The Millennium Institute for Vaccine Development and Technology (CNPq - 420067/2005-1), CNPq and FAPEMIG supported this work.

\section{References}

[1] G. Grimaldi Jr., R.B. Tesh, Leishmaniasis of the New World: current concepts and implications for future research, Clin. Microbiol. Rev. 6 (1993) 230-250.

[2] G. Grimaldi Jr., R.B. Tesh, D. McMahon-Pratt, A review of the geographic distribution and epidemiology of leishmaniasis in the New World, Am. J. Trop. Med. Hyg. 41 (1989) 687-725. 
[3] S. Gurunathan, C. Prussin, D.L. Sacks, R.A. Seder, Vaccine requirements for sustained cellular immunity to an intracellular parasitic infection, Nat. Med. 4 (1998) 1409-1415.

[4] E. Dumonteil, F. Andrade-Narvarez, J. Escobedo-Ortegon, M.J. RamirezSierra, G. Valencia-Pacheco, A. Flores-Serrano, S. Canto-Lara, A. Arjona-Torres, Comparative study of DNA vaccines encoding various antigens against Leishmania mexicana, Dev. Biol. 104 (2000) 135-141.

[5] E.A. Coelho, C.A. Tavares, F.A. Carvalho, K.F. Chaves, K.N. Teixeira, R.C. Rodrigues, H. Charest, G. Matlashewski, R.T. Gazzinelli, A.P. Fernandes, Immune responses induced by the Leishmania (Leishmania) donovani $\mathrm{A} 2$ antigen, but not by the LACK antigen, are protective against experimental Leishmania (Leishmania) amazonensis infection, Infect. Immun. 71 (2003) 3988-3994.

[6] E. Dumonteil, R.S. Maria Jesus, E.O. Javier, G.M. Del Rosário, DNA vaccines induce partial protection against Leishmania mexicana, Vaccine 21 (2003) 2161-2168.

[7] H. Charest, G. Matlashewski, Developmental gene expression in Leishmania donovani: differential cloning and analysis of an amastigotestage-specific gene, Mol. Cell. Biol. 14 (1994) 2975-2984.

[8] E. Ghedin, W.W. Zhang, H. Charest, S. Sundar, R.T. Kenney, G. Matlashewski, Antibody response against a Leishmania donovani amastigote-stage-specific protein in patients with visceral leishmaniasis, Clin. Diagn. Lab. Immunol. 4 (1997) 530-535.

[9] A. Ghosh, S. Labrecque, G. Matlashewski, Protection against Leishmania donovani infection by DNA vaccination: increased DNA vaccination efficiency through inhibiting the cellular p53 response, Vaccine 19 (2001) 3169-3178.

[10] A. Ghosh, W.W. Zhang, G. Matlashewski, Immunization with A2 protein results in a mixed $\mathrm{Th} 1 / \mathrm{Th} 2$ and a humoral response which protects mice against Leishmania donovani infection, Vaccine 20 (2002) 59-66.

[11] C.B. Palatnik-de-Sousa, H.S. Dutra, R. Borojevic, Leishmania donovani surface glycoconjugate GP36 is the major immunogen component of the Fucose-Mannose Ligand (FML), Acta Trop. 53 (1993) 59-72.

[12] W. Shi, V.L. Scharamm, S.C. Almo, Nucleoside hydrolase from Leishmania major. Cloning, expression, catalytic properties, transition states inhibitors, and the 2.5-a crystal structure, J. Biol. Chem. 274 (1999) 21114-21120.

[13] E. Paraguai-de-Souza, R.R. Bernardo, M. Palatnik, C.B. Palatnik-deSousa, Vaccination of Balb/c mice against experimental visceral leishmaniasis with the GP36 glycoprotein antigen of Leishmania donovani, Vaccine 19 (2001) 3104-3115.

[14] I. Aguilar-Be, R.S. Zardo, E. Paraguai-de-Souza, G.P. Borja-Cabrera, M. Rosado-Vallado, M. Mut-Martin, M.R. Garcia-Miss, C.B. Palatnikde-Sousa, E. Dumonteil, Cross-protective efficacy of a prophylactic Leishmania donovani DNA vaccine against visceral and cutaneous murine leishmaniasis, Infect. Immun. 73 (2005) 812-819.

[15] S. Gurunathan, C. Wu, B.L. Freidag, R.A. Seder, DNA vaccines: a key for inducing long-term cellular immunity, Curr. Opin. Immunol. 12 (2000) 442-447.
[16] K. Ito, K. Ito, N. Shinohara, S. Kato, DNA immunizaton via intramuscular and intradermal routes using a gene gun provides different magnitudes and durations on immune response, Mol. Immunol. 39 (2003) 847-854.

[17] M.M. Bradford, A rapid and sensitive method for the quantification of microgram quantities of protein utilizing the principle of protein-dye binding, Anal. Biochem. 72 (1976) 248-254.

[18] E.A. Marques-da-Silva, E.A.F. Coelho, D.C.O. Gomes, M.C. Vilela, C.Z. Masioli, C.A.P. Tavares, A.P. Fernandes, L.C.C. Afonso, S.A. Rezende, Intramuscular immunization with p36 (LACK) DNA vaccine induces IFN- $\gamma$ production but does not protect BALB/c mice against Leishmania chagasi intravenous challenge, Parasitol. Res. 98 (2005) 67-74.

[19] F.A.A. Carvalho, H. Charest, C.A.P. Tavares, G. Matlashewski, E.P. Valente, A. Rabello, R.T. Gazzinelli, A.P. Fernandes, Diagnosis of American visceral leishmaniasis in humans and dogs using the recombinant Leishmania donovani A2 antigen, Diag. Microbiol. Infect. Dis. 43 (2002) 289-295.

[20] L.C. Afonso, P. Scott, Immune responses associated with susceptibility of C57BL/10 mice to Leishmania amazonensis, Infect. Immun. 61 (1993) 2952-2959.

[21] P.C. Melby, Y.Z. Yang, J. Cheng, W. Zhao, Regional differences in the cellular immune response to experimental cutaneous or visceral infection with Leishmania donovani, Infect. Immun. 66 (1998) 18-27.

[22] J. Lehmann, K.H. Enssle, I. Lehmann, A. Emmendorfer, M.L. LohmannMatthes, The capacity to produce IFN-gamma rather than the presence of interleukin-4 determines the resistance and the degree of susceptibility to Leishmania donovani infection in mice, J. Interferon Cytokine Res. 20 (2000) 63-77.

[23] J. Ji, J. Sun, L. Soong, Impaired expression of inflammatory cytokines and chemokines at early stages of infection with Leishmania amazonensis, Infect. Immun. 71 (2003) 4278-4288.

[24] M.E. Wilson, M. Sandor, A.M. Blum, B.M. Young, A. Metwali, D. Elliott, R.G. Lynch, J.V. Weinstock, Local suppression of IFN-gamma in hepatic granulomas correlates with tissue-specific replication of Leishmania chagasi, J. Immunol. 156 (1996) 2231-2239.

[25] M.E. Wilson, S.M. Jeronimo, R.D. Pearson, Immunopathogenesis of infection with the visceralizing Leishmania species, Microb. Pathog. 38 (2005) $147-160$.

[27] U.M. Padigel, J. Alexander, J.P. Farrell, The role of interleukin-10 in susceptibility of BALB/c mice to infection with Leishmania mexicana and Leishmania amazonensis, J. Immunol. 171 (2003) 3705-3710.

[28] N.B. Norsworthy, J. Sun, D. Elnaiem, G. Lanzaro, L. Soong, Sand fly saliva enhances Leishmania amazonensis infection by modulating Interleukin-10 production, Infect. Immun. 72 (2004) 1240-1247.

[29] C.B. Stober, U.G. Lange, M.T. Roberts, A. Alcami, J.M. Blackwell, IL10 from regulatory $\mathrm{T}$ cells determines vaccine efficacy in murine Leishmania major infection, J. Immunol. 175 (2005) 2517-2524.

[30] A.C. Stanley, C.R Engwerda, Balancing immunity and pathology in visceral leishmaniasis, Immunol. Cell Biol. 85 (2007) 138-147. 